\title{
Molecular Diagnosis of Thyroid Nodules and Its Future Implications for the Management of Thyroid Cancer
}

\author{
Rodrigo Arrangoiz ${ }^{1 *}\left(\mathbb{D}\right.$, Jeronimo Garcialopez De Llano $^{1}$, Maria Fernanda Mijares ${ }^{1}$, \\ Gonzalo Fernandez-Christlieb ${ }^{2}$, Vanitha Vasudevan' ${ }^{1}$, Amit Sastry ${ }^{1}$, Adrian Legaspi ${ }^{1}$, \\ Jennifer Fernandez ${ }^{1}$, Frank de la Cruz ${ }^{1}$, Fernando Cordera ${ }^{2}$, Daniel Margain ${ }^{1}$ \\ ${ }^{1}$ Center for Advanced Surgical Oncology, Steward Health, Hialeah, FL, USA \\ ${ }^{2}$ American British Cowdray Medical Center, Mexico City, Mexico \\ Email: ^rodrigo.arrangoiz@steward.org, jerogldll@gmail.com, mfmo.md@gmail.com, g.fernandez@mail.harvard.edu, \\ vanitha.vasudevan@tenethealth.com, amit.sastry@tenethealth.com, adrian.legaspi@tenethealth.com, \\ jennifernandez.g24@gmail.com, frankdlc@sanjuanbautista.edu,fernando.cordera@gmail.com, \\ dmargaint@gmail.com
}

How to cite this paper: Arrangoiz, R., De Llano, J.G., Mijares, M.F., Fernandez-Christlieb, G., Vasudevan, V., Sastry, A., Legaspi, A., Fernandez, J., de la Cruz, F., Cordera, F. and Margain, D. (2021) Molecular Diagnosis of Thyroid Nodules and Its Future Implications for the Management of Thyroid Cancer. International Journal of Otolaryngology and Head \& Neck Surgery, 10, 398418.

https://doi.org/10.4236/ijohns.2021.105037

Received: August 9, 2021

Accepted: September 10, 2021

Published: September 13, 2021

Copyright $\odot 2021$ by author(s) and Scientific Research Publishing Inc. This work is licensed under the Creative Commons Attribution International License (CC BY 4.0)

http://creativecommons.org/licenses/by/4.0/ (c) (i) Open Access

\begin{abstract}
Molecular testing in thyroid nodules and thyroid cancer is rapidly evolving; care must be used when incorporating molecular testing for thyroid nodules into clinical practice. A clear appreciation of the goals and restraints of molecular testing must be integrated into how physicians use and explain molecular testing to patients. Molecular tests can help rule in cancer for indeterminate thyroid nodules with very specific mutations for thyroid cancer, such as BRAF and RET/PTC, and can help reduce the rates of completion thyroidectomies in this era of de-escalation of the management of thyroid disease. The positive predictive value (PPV) of malignant cytology (Bethesda VI) is 98\%; and even though molecular testing improves specificity and PPV, it falls short of this ideal for other mutations. We present a detailed evaluation of the current state of molecular testing and their clinical relevance in the setting of diagnostic utility and their impact on surgical decision-making. By recapitulating the clinical impact of these tests and some of the related drawbacks, we hope to provide adequate up to date information of the appropriate utilization of these tools in the management of indeterminate or suspicious thyroid nodules and highlight future directions on their utilization for the management of thyroid cancer.
\end{abstract}

\section{Keywords}

Thyroid Cancer, Papillary Thyroid Cancer, Papillary Thyroid 
Microcarcinoma, Thyroid Nodule, Thyroid Cancer Treatment, Molecular Studies for Thyroid Cancer, Affirma, Thyroseq

\section{Introduction}

Approximately $3.4 \%$ of all cancer diagnosis each year are thyroid cancers (TC), making it the most common endocrine malignancy [1]. The most accepted theory of thyroid follicular cell carcinogenesis involves a multistep process that leads to the transformation of the thyroid follicular cells resulting in differentiated or undifferentiated TC. Well-defined molecular aberrations have been linked with specific TC stages, propelling the progression from well-differentiated to undifferentiated follicular-derived thyroid carcinomas [2]. In recent times, the cancer stem-like cell theory has been suggested, in which phenotypically diverse cancer cells could be spawned by a small subpopulation of stem cells after genetic and epigenetic transformations [3].

In the past three decades, the accessibility to the genome sequence has yielded much progress in clarifying the molecular mechanisms underlying TC [4]. Genetically TC is an extremely simple disease with a low burden of somatic mutations in each tumor [2]. Mutations that offer a selective growth advantage known as driver mutations are found in more than $90 \%$ of TC, helping to promote the development of this cancer [2] [4]. The molecular pathogenesis of the majority of TC involves dysregulation of the mitogen-activated protein kinase (MAPK) and phosphatidylinositol-3 kinase (PI3K)/AKT signaling pathways. MAPK activation is considered to be crucial for PTC initiation, through point mutations of the BRAF and RAS genes or gene fusions of RET/PTC and TRK. On the other hand, PI3K/AKT activation is thought to be critical in FTC initiation and can be triggered by activating mutations in RAS, PIK3CA, and AKT1 as well as by inactivation of PTEN, which negatively regulates this pathway. TC progression and dedifferentiation to PDTC and ATC involves a number of additional mutations affecting other cell signaling pathways, such as p53 and $\mathrm{Wnt} / \beta$-catenin. More recently, TERT promoter mutations have been described in all the histological TC type, with a significantly higher prevalence in aggressive and undifferentiated tumors, indicating their role in TC progression. Mutations in the RET (Rearranged during transfection) proto-oncogene account for most MTC cases and can occur sporadically or as inherited germline events in the multiple endocrine neoplasia type 2A (MEN2A) and 2B (MEN2B) syndromes. A minority of sporadic MTC are caused by $\mathrm{H}-, \mathrm{K}-$, and N-RAS mutations.

Thyroid nodules are a frequent problem in every day clinical practice, with studies suggesting that nearly two-thirds of the general population harbor thyroid nodules when evaluated by ultrasound imaging [5] [6]. The prevalence rates of thyroid nodules in the adult population are around 70\% [7]. Eighty to $90 \%$ of thyroid nodules are benign, and the ability to be able to detect malignancy and 
establish the appropriate management is of paramount importance [7]. The malignancy potential of a thyroid nodule is determined in a multimodal fashion that includes a good history, physical examination, imaging evaluation, and fine needle aspiration (FNA) [6]-[11]. Cytopathologic analysis of FNAs has surfaced as a key adjunct to the clinical and imaging measures used to characterize thyroid nodules. In 2009 the Bethesda System for Reporting Thyroid Cytopathology (TBSRTC) was developed by the National Cancer Institute, and it was subsequently revised in 2017 [12] [13]. It is used extensively to stratify the risk of malignancy based on cytopathology and has been shown to accurately corroborate the diagnosis between benign versus malignant nodules in $70 \%$ to $80 \%$ of the cases [12] [13]. Nonetheless, this still leaves $20 \%$ to $30 \%$ of FNA cases to be categorized as indeterminate or suspicious (Bethesda III, IV, and V) [6] [12] [13]. Thyroid nodules categorized as indeterminate or suspicious have been shown to have malignancy rates ranging from $6 \%$ to $75 \%$ on final pathology [6] [12] [13]. Innovative tools are being developed to help characterize the malignant potential of indeterminate and suspicious thyroid nodules. Molecular testing for thyroid nodules has advanced very quickly over the past decade both to help improve the diagnostic accuracy of thyroid cytology for indeterminate cases and potentially to guide the extent of surgery as initial therapy for suspected thyroid malignancies.

Molecular markers can be categorized based on their intended use; that is, for diagnostic purposes (classification of a disease state), prognostic purposes, or predictive purposes (providing information on the estimated probability of therapeutic benefit or harm of a specific therapy) [14]. Additionally, predictive molecular markers recognize subgroups of patients in which a therapeutic intervention may be proven to be either beneficial or harmful, with implications for proper clinical stratification of management options [14]. Studies that validate molecular marker tests include assessment of analytic validity (test accuracy and reproducibility), clinical validity (performance of the test in distinguishing different groups of patients, based on biology or expected disease outcome, including sensitivity and specificity or predictive values), and clinical utility (assessment of the test's ability to improve outcomes, with direct clinical decision-making implications) [14].

Molecular testing has become a common tool in clinical practice for thyroid nodule evaluation due to the recent improvements in the comprehension of the molecular foundations and genotype-phenotype parallels in oncology. For example, the detection of the oncogenic BRAF V600E mutation in roughly $50 \%$ to $70 \%$ papillary thyroid cancers (PTC) and its related susceptibility to BRAF targeted therapies have dramatically shifted the landscape of PTC management [15]. In the last couple of years multi-gene panel molecular tests that intend to improve the diagnostic accuracy in indeterminate and suspicious nodules (Bethesda III to IV) have emerged as part of the algorithm in the work-up and management of thyroid nodules and cancer [6] [7]. Two of the most extensively studied multi-gene panel molecular tests are the Afirma Gene Expression Classifier 
(GEC) which evaluates $m R N A$ expression levels of 167 genes and the ThyroSeq v2, which evaluates DNA mutations within a 19 gene panel [16]. These two tests have been updated to the Afirma Genomic Sequencing Classifier (GSC) based on RNA sequencing technology evaluating roughly 10,000 genes and the ThyroSeq v3 next generation sequencing of 112 genes [16] [17] [18]. Other tests available commercially include assays of oncogenic microRNA expression (e.g., Rosetta GX Reveal, ThyraMIR), and a seven-gene panel test for oncogenic point mutations and gene fusions [19] [20] [21]. These molecular tests are now part of standard of care for the diagnostic evaluation of thyroid nodules with multiple medical societies recommending molecular testing as diagnostic adjuncts for indeterminate or suspicious thyroid nodules [7] [9] [22] [23].

We present a detailed evaluation of the current state of molecular testing and their clinical relevance in the setting of diagnostic utility and their impact on surgical decision-making. By recapitulating the clinical impact of these tests and some of the related drawbacks, we hope to provide adequate up to date information of the appropriate utilization of these tools in the management of indeterminate or suspicious thyroid nodules and highlight future directions on their utilization for the management of thyroid cancer.

\section{Molecular Alterations in Thyroid Cancer}

The main types of mutations identified in the two most common subtypes of differentiated thyroid cancer, PTC and follicular thyroid carcinoma (FTC), are BRAF and RAS point mutations, RET/PTC, and PAX8/peroxisome proliferator-activated receptor $\gamma(\operatorname{PPAR} \gamma)$ rearrangements [24]. Currently, these mutations convey the greatest impact on tumor diagnosis and prognostication. PTCs contain point mutations of the BRAF and RAS genes and RET/ PTC rearrangements, all of which can activate the mitogen-activated protein kinase (MAPK) pathway [24]. These reciprocally exclusive mutations are identified in greater than 70\% of PTC [25] [26] [27] [28]. FTCs contain either RAS mutations or PAX8/PPAR $\gamma$ rearrangement [24]. These mutations are likewise reciprocally exclusive and are found in roughly $75 \%$ of FTC [29].

One more type of genetic aberration identified in patients with thyroid cancer involves the PI3K/AKT signaling pathway, which rare in well-differentiated thyroid cancers, but they have a greater prevalence in less-differentiated thyroid cancers [30] [31] [32]. Other mutations known to occur in poorly differentiated and anaplastic carcinomas include the TP53 and CTNNB1 genes [33]. Still, these mutations are infrequent in well-differentiated PTC or FTC [24]. Roughly $5 \%$ of PTC harbor TRK rearrangement, the prevalence of this mutation is extremely low, especially in North America [34] [35].

\section{Shortcomings of Thyroid Cytopathologic Evaluation}

Imaging with high resolution ultrasound and ultrasound-guided FNA has long been the most common method of the evaluation of thyroid nodules [36]. Even though it is extremely accurate for the diagnosis of benign colloid nodules (the 
most common finding) and classic PTC, thyroid FNA is much less accurate for the diagnosis of follicular variant of PTC [6] [7]. Moreover, the ability to discriminate between follicular (or Hurthle cell) adenomas and carcinomas necessitates histologic assessment for capsule or vascular invasion and therefore cannot be diagnosed with cytopathology alone. Additionally, numerous benign ailments pose cytologic challenges, such as hyperplastic adenomatoid nodules, and inflammatory conditions, such as lymphocytic or granulomatous thyroiditis [37]. In many circumstances, thyroid nodule FNA results are cytologically indeterminate or suspicious ( $20 \%$ to $30 \%$ of the time) and historically have necessitated diagnostic thyroid surgery, primarily thyroid lobectomy, for a definitive diagnosis [37].

To tackle this variability in the reporting and classification of cytologic findings, the Bethesda system was published in 2009 and subsequently revised in 2017 [12] [13]. The Bethesda system had been broadly accepted and has clearly shown to improve the reliability of the terminology regarding the potential risk of malignancy of indeterminate nodules, but the rates of malignancy within the subcategories can vary extensively by institution. Additionally, noteworthy interobserver and intraobserver variability exist in the interpretation of FNA cytology, with concordance rates of $65 \%$ to $75 \%$ for thyroid cytology and $90 \%$ for histopathology [38]. Molecular testing of thyroid nodules has the possibility to help differentiate indeterminate or suspicious FNA results as either benign or neoplastic, therefore improving the quality of care and guide surgical decisionmaking [37]. Nevertheless, the assimilation of molecular testing into the algorithm for diagnosis in thyroid cancer may influence variations in cytopathology interpretation leading to modifications in the rates of indeterminate or suspicious thyroid nodules [39].

Even final pathologic assessment of benign versus malignant disease is in evolution, as exemplified by the changes in the nomenclature when discussing noninvasive, encapsulated follicular variant of PTC, which was modified to be considered nonmalignant and redefined as a noninvasive follicular thyroid neoplasm with papillary-like features (NIFTP) [40]. Nonetheless, diagnostic hemithyroidectomy is still essential for both pathologic diagnosis and treatment of NIFTP [40]. This is comparable to differentiating between follicular adenoma and follicular carcinoma, in which pathologic assessment is necessary, to exclude capsular or vascular invasion in the diagnosis of follicular carcinoma [37].

\section{Indeterminate Thyroid Nodules (Bethesda III, IV, V)-Table 1}

1) Atypia of Undetermined Significance (AUS) or Follicular Lesion of Undetermined Significance (FLUS) on Cytology

Based on the Bethesda system, this diagnostic category is reserved for specimens that contain cells with architectural and/or nuclear atypia that are more prominent than expected for benign changes, but not sufficient to be placed in one of the highest-risk diagnostic categories [8] [13]. In the studies that used the 
Table 1. The 2017 Bethesda system for reporting thyroid cytopathology [13].

\begin{tabular}{cccc}
\hline Diagnostic Category & $\begin{array}{c}\text { Risk of Malignancy if } \\
\text { NIFTP Not Cancer }\end{array}$ & $\begin{array}{c}\text { Risk of Malignancy if } \\
\text { NIFTP Equals Cancer }\end{array}$ & Usual Management \\
\hline Non-diagnostic or unsatisfactory & $5 \%$ to $10 \%$ & $5 \%$ to $10 \%$ & $\begin{array}{c}\text { Repeat FNA with ultrasound guidance } \\
\text { Clinical and sonographic follow-up }\end{array}$ \\
Benign & $0 \%$ to $3 \%$ & $0 \%$ to $3 \%$ & Repeat FNA, molecular testing, or lobectomy \\
$\begin{array}{c}\text { Atypia of undetermined significance or } \\
\text { follicular lesion of undetermined significance }\end{array}$ & $6 \%$ to $18 \%$ & $10 \%$ to $30 \%$ & Molecular testing, or lobectomy \\
$\begin{array}{c}\text { Follicular neoplasm or suspicious } \\
\text { for a follicular neoplasm }\end{array}$ & $10 \%$ to $40 \%$ & $25 \%$ to $40 \%$ & Near-total thyroidectomy or lobectomy \\
$\quad$ Suspicious for malignancy & $45 \%$ to $60 \%$ & $50 \%$ to $75 \%$ & Near-total thyroidectomy or lobectomy \\
Malignant & $94 \%$ to $96 \%$ & $97 \%$ to $99 \%$ &
\end{tabular}

criteria established by the Bethesda system, the risk of cancer for patients with AUS/FLUS who underwent surgery was $6 \%$ to $18 \%$ if NIFT (non-invasive follicular thyroid neoplasia with papillary nuclear characteristics) it is not considered cancer, and $10 \%$ to $30 \%$ if NIFT is considered a cancer [24].

For thyroid nodules with AUS/FLUS cytology after a FNAB, with clinical and ultrasonographic features of concern, the assessment can be continued by repeating the FNAB or if the technology is available, molecular tests can be used to complement the risk assessment of malignancy instead of preceding directly with a strategy of either surveillance or diagnostic surgery (lobectomy). Patient preference should be considered in decision-making (recommendation 15 of the ATA) [9]. If FNAB is not repeated, and molecular tests are not performed, or both studies were inconclusive, a diagnostic surgical excision may be performed for the thyroid nodules with Bethesda AUS/FLUS classification, according to the clinical risk factors, the ultrasonographic pattern, and patient preference (recommendation 15 of the ATA) [7].

2) Folicular Neoplasm/Suspicious Folicular Neoplasm Cytology (FN/SFN)

This diagnostic category of the Bethesda system is used for cellular aspirates:

- Composed by follicular cells arranged in an altered architectural pattern characterized by cell crowding and/or microfollicular formation, lacking nuclear characteristics of papillary carcinoma

or

- Composed almost exclusively oncocytic cells (Hurthle).

This is a category has an intermediate risk of malignancy in the Bethesda system, with an estimated risk of malignancy between $10 \%$ to $40 \%$ if NIFT is not considered cancer and between $25 \%$ to $40 \%$ if NIFT is considered cancer [9] [30]. This category represents $1 \%$ to $25 \%$ (average, $10 \%$ ) of all FNA samples [9].

Diagnostic surgical excision (lobectomy) is the long-established standard for the treatment of thyroid nodules with a FN/SFN cytology. However, if you have the technology, after considering the clinical assessment and the ultrasonographic characteristics, molecular tests can be used to complement the assessment of the 
risk of malignancy instead of proceeding directly with surgery (recommendation 16) of the ATA) [7]. Patient preference should be considered in clinical decision making. If the molecular tests cannot be performed or are indeterminate, surgical excision can be considered for the definitive diagnosis of thyroid nodules classified as FN/SFN (recommendation 16 of the ATA) [7].

3) Suspicious Cytology for Malignancy

This diagnostic category of the Bethesda system represents $1 \%$ to $6 \%$ of all FNABs and is reserved for aspirates with cytological features that generate a high suspicion of malignancy (mainly for papillary thyroid carcinoma) but that are not sufficient for a conclusive diagnosis [12] [13] [41] [42]. This is the highest risk category for indeterminate cytology in the Bethesda System, with an estimated cancer risk of $45 \%$ to $60 \%$ if NIFT is not considered cancer and $50 \%$ to $75 \%$ if NIFT is considered cancer [13]. Due to the high risk of cancer, the diagnosis of suspicious papillary carcinoma is an indication for surgery [7].

If FNAB results in a suspicious cytology for papillary thyroid carcinoma, the surgical treatment should be very similar to the management of a frankly malignant reported for the FNA. Factors that we must take into account in offering the definitive treatment with a suspicious cytology for papillary thyroid carcinoma, are the clinical risk factors, the ultrasonographic characteristics, the patient's preference, and possibly the results of the molecular tests (BRAF, RAS, RET/PTC, PAX8/PPAR) (recommendation 17 of the ATA) [7].

\section{Development of Thyroid Molecular Testing}

Immunohistochemical assessment with Galectin-3 and additional biomarkers exhibited some potential to enhance the diagnostic precision of thyroid cytology, but they did not have adequate reproducibility and sensitivity to consistently exclude malignancy. Researchers pursued the identification of specific gene mutations and gene rearrangements that were pathogenic in thyroid cancer. Molecular testing for known DNA and RNA mutations have been shown to improve the diagnostic accuracy of FNA biopsy when added to cytologic evaluation [37]. Roughly $70 \%$ of thyroid carcinomas harbor the following DNA and RNA mutations: the Harvey rat sarcoma viral oncogene homolog (H-Ras), neuroblastoma rat sarcoma viral oncogene homolog (N-Ras), Kirsten rat sarcoma viral oncogene homolog (K-Ras), rearranged during transfection/PTC 1 (Ret/PTC1), Ret/PTC2, Ret/PTC3, v-Raf murine sarcoma viral oncogene homolog $B 1$ [BRAF], and paired box gene 8-peroxisome proliferator-activator receptor c (Pax8-PPARc) [37]. The latter mutations identified enhance the specificity of FNA cytology, but they are not as sensitive as a detection tool to rule out cancer for cases in which no mutation is identified [37].

Tackling the question of benign versus malignant etiology from a another standpoint, a gene expression classifier (GEC) was developed through an iterative process that intended for the test to have a high sensitivity and a high negative predictive value (NPV), analogous to the NPV of thyroid nodules diag- 
nosed as benign on cytology [43] [44]. Intended specifically to aid in ruling out malignancy, the Afirma GEC was developed to possibly decrease the rate of diagnostic lobectomies in the case of indeterminate or suspicious thyroid nodules [37].

Furthermore, as molecular testing was been established for clinical use to help enhance the diagnostic accurateness of thyroid nodule FNA, The Cancer Genome Atlas (TCGA) project recorded the mutations attributed to both classic and follicular variants of PTC, considerably decreasing the number of unidentified mutations instigating differentiated thyroid cancer [45]. Integrating the new data obtained through TCGA, an updated gene panel using next-generation sequencing (NGS) was developed for clinical use ThyroSeq NGS, v2 [46] [47] [48]. The ThyroSeq NGS v2 was developed to enhance the overall accuracy of molecular testing to distinguish between both benign and malignant thyroid cytopathology by improving sensitivity and NPV with only a mild reduction in specificity and PPV [46] [47] [48].

Further methods to molecular testing for thyroid cytopathology include the development of micro-RNA (miRNA) testing. MiRNAs act as negative regulators of gene expression and may influence cellular processes that lead to carcinogenesis. Since miRNA expression may be dysregulated in thyroid cancer, this is an extra tactic to molecular test development with high sensitivity and high specificity [5] [49] [50].

\section{Gene Mutation Panel: A “Rule-In” Test}

When an indeterminate or suspicious thyroid nodule with a specific gene mutation that correlates with a high risk of malignancy, a thyroid cancer may be diagnosed before surgery and successfully limit the number of patients who require a two-part operation for definitive management of the cancer. A rule-in test is of value when it changes clinical care, such as altering the extent of thyroid surgery from a lobectomy to a total thyroidectomy [7]. The seven-gene specific mutation panel has been proven to be an effective "rule-in" cytomolecular test for thyroid malignancy based on the high specificity and PPV, but this test has not been deemed as an effective "rule-out" test because of low sensitivity [37]. A large prospective, single-institution study with both cytology and histologic follow-up encompassed 247 Bethesda III nodules and 214 Bethesda IV nodules. The researchers reported a $63 \%$ sensitivity and $99 \%$ specificity for Bethesda III nodules and $57 \%$ sensitivity and $97 \%$ specificity for Bethesda IV nodules. The PPV (a positive test result) increased the risk of malignancy to $88 \%$ for Bethesda III nodules and $87 \%$ for Bethesda IV nodules, whereas the absence of a mutation or fusion was associated with a cancer risk of $6 \%(\mathrm{NPV}=94 \%)$ and $14 \%(\mathrm{NPV}=$ $86 \%)$, respectively [50].

Even though the early acceptance of the 7-gene mutation panel to help guide the extent of the operation was very positive [50], with a substantial reduction in the number of patients necessitating a completion thyroidectomy, a change in 
the pendulum in the management of thyroid cancer has limited this early promise. The 2015 American Thyroid Association guidelines have recommended a more judicious approach to thyroid surgery [7], identifying that a total thyroidectomy may not be the initial recommended management option even for corroborated thyroid malignancies that are clinically considered low risk. Initial research with the 7-gene mutation panel indicated that rat sarcoma (RAS) (a family of small guanosine triphosphate hydrolases) mutations were most common but were not highly specific for thyroid cancer, thereby limiting the PPV of the test [37]. Multiple studies have established that RAS mutations also can be recognized in up to $48 \%$ of benign thyroid nodules; consequently, the type of RAS mutation and the degree of allelic frequency can be used to guide decision-making [51]. Likewise, numerous thyroid nodules with RAS and PAX8/PPARc mutations are noninvasive encapsulated follicular variant of PTC [52], now reclassified as NIFTP [40]. The term NIFTP was introduced to describe a slow growing and well-circumscribed case of follicular variant papillary thyroid cancers that unlike other forms of PTC, NIFTP tumors do not seem to grow or spread, and therefore may be able to be treated like benign thyroid nodules [40]. Nevertheless, treatment by lobectomy is still recommended [40] [52]. The 2015 American Thyroid Association guidelines do not dictate a total thyroidectomy for differentiated thyroid cancer less than $4 \mathrm{~cm}$ with any of the mutations found in the 7-gene mutation panel, further constraining its usefulness in either decreasing excessive surgery or guiding the extent of surgery for patients with indeterminate nodules [43].

\section{Afirma GEC: A “Rule-Out" Test}

A test with a high sensitivity and high negative predictive value (NPV) can ruleout cancer [53] [54]. A test sensitivity measures the fraction of cancers that the test identifies as "positive" (Afirma GEC suspicious). The Afirma GEC sensitivity among indeterminate nodules is roughly $90 \%$ [44]. A test NPV measures the fraction of "negative" calls by the test (Afirma GEC benign) that are correct. The Afirma GEC NPV is $94 \%$ to $95 \%$ amongst Bethesda III and IV nodules at a cancer prevalence of $24 \%$ to $25 \%$ [44]. Although not mutually exclusive, a test with a high specificity and high PPV is able to rule-in cancer. A test specificity measures the fraction of benign nodules that are called benign by the test. The Afirma GEC test specificity is 52\% [44], signifying that just over half of the benign nodules are called GEC benign. A test PPV measures the fraction of "positive" calls by the test (Afirma GEC suspicious) that are correct. The Afirma GEC test PPV is $37 \%$ to $38 \%$ amid Bethesda III and IV nodules [44]. Accordingly, the strength of the Afirma GEC is its ability to rule-out cancer (NPV), more than its ability to rule-in cancer (PPV).

As mentioned previously a rule-in test is of significance when it changes clinical care, such as altering the extent of thyroid surgery from a lobectomy to a total thyroidectomy. Hence, the effectiveness of a rule-in tests is currently ques- 
tioned as patient benefit has not been established. Given the low specificity (52\%) and PPV (37\% to 38\%) of Afirma, it is not considered a rule-in test. Even though an Afirma GEC suspicious result raises the risk of cancer from $24 \%$ to $25 \%$ to $37 \%$ to $38 \%$, it should be clear that the asset of the test is that it finds just over one-half of all benign nodules with Bethesda III or IV cytology as genomically benign, and $90 \%$ of all cancers as genomically suspicious irrespective of the cancer prevalence. Therefore, when utilized as part of the diagnostic armamentarium for cytologically indeterminate nodules with a risk of malignancy of $25 \%$ or less, the estimated accuracy of a benign result (NPV) is $94 \%$ or greater. Accordingly, the majority of the Afirma GEC benign nodules are entrants for clinical observation instead of a diagnostic operation. Rare neoplasms that are often difficult to accurately diagnose with cytology such as parathyroid neoplasms, medullary thyroid carcinomas (MTC), and metastases to the thyroid from malignant breast, melanoma, and renal cell carcinomas are easily identified by the Afirma GEC. Once the test fails to identify one of these rare tumors, the GEC evaluates the expression of 142 genes that are used in a proprietary mathematical algorithm to categorize indeterminate thyroid nodule as either GEC benign or GEC suspicious.

The Afirma GEC is founded on the quantification of messenger ribonucleic acid (mRNA) expression. There are quite a few diagnostic benefits to using RNA instead of other approaches such as microRNA expression or DNA mutations [55]. The BRAF V600E mutation which is the most common genomic alteration identified in differentiated thyroid cancers, it is usually not identified in cytologically indeterminate thyroid nodules (Bethesda III and IV). The most common mutation among indeterminate thyroid nodules are RAS mutations, but these are identified in both malignant and benign nodules. Since benign nodules outnumber malignant nodules 4:1 among indeterminate nodules, the PPV of RAS mutations is poor in a multiple of studies [56]-[61]. The difficulties of using mutational approaches for indeterminate or suspicious thyroid nodules is that many malignancies do not have the known genomic aberrations, and when present, most genomic anomalies are not specific for cancer [62].

In the development of the Afirma GEC, instead of discriminately relying on genes formerly detected in the medical literature, analysis of the whole genome (transcriptome) was used to isolate candidate genes, and support vector machine learning methods were used to develop the classifier algorithm [44] [63].

The clinical study validating the Afirma GEC was originally performed on a small independent sample of thyroid nodule FNABs within a prospective multicenter, double blind study design [63]. The Afirma GEC accomplished a high sensitivity and NPV, including among indeterminate thyroid nodules. The GEC was validated in a second larger independent sample in a prospective multicenter study which included the largest ever prospectively collected set of thyroid FNAB biopsies from 3789 unique patients, with a final validation set of 265 indeterminate nodules [44]. Based on the $24 \%$ prevalence of malignancy in cyto- 
logically indeterminate samples (Bethesda III/IV), a 95\% NPV for the Afirma GEC was attained [44].

The distinctive and often unnoticed strength of this prospective, multicenter, and blinded validation design is that it supports generalizability of the results. The prospective and multicenter study design reduces selection bias and enhances what is likely to occur in the real-world. The patients (3789) were prospectively consented and enrolled in the trial before undergoing FNAB at 49 study sites, including academic and community practices, which offers confidence in the external validity of the findings. A strong internal validity was established when no differences were found between the final validation cohort of 265 patients compared to the full prospective and consecutive total enrollment cohort. The investigators were blinded to the Afirma GEC results, impeding them from being influenced in the decision of which surgery should be recommended. These vital study design elements support the internal and external validity of the Afirma GEC study and provide confidence in the broader generalizability of the study findings to every day clinical practice [7].

The pre-test risk of malignancy (ROM) determines the PPV and NPV of the Afirma GEC. To practice personalized medicine, it is central to consider the individual patient's pre-test risk. The patient's pre-test ROM includes their individual characteristics (gender, history of childhood radiation, imaging findings, serum TSH among others) and the interpreting cytopathologist's thresholds to use cytology indeterminate categories. Neglecting this very vital step of personalized care and believing that every patient has the same pre-test risk overlooks very important medical information.

The ATA guidelines allow for either thyroid lobectomy or near-total/total thyroidectomy for thyroid malignancy $1 \mathrm{~cm}$ to $4 \mathrm{~cm}$ in size without gross extra-thyroidal extension or clinical evidence of lymph node metastases [7]. Therefore, numerous factors must be taken into consideration when planning the operation for indeterminate thyroid nodules, such as the risks and benefits, the presence of significant contralateral nodules, long-term follow-up, the role for completion thyroidectomy with or without radioactive iodine ablation if malignancy is found, and patient wishes.

The 2015 ATA guideline emphasizes ultrasound characteristics to predict the nodule's ROM [7]. The Afirma GEC is anticipated to identify $90 \%$ of cancers (sensitivity) as GEC suspicious, and 52\% of the benign nodules as GEC benign (specificity), regardless of the pre-test ROM. High suspicion ultrasound patterns may be related with a greater than $70 \%$ ROM and are found in the minority of nodules with indeterminate cytology [58] [59] [61] [62] [63] [64]. Thyroid nodules with such a high pre-test ROM, the NPV of Afirma is expected to be less than $70 \%$, so it may not be useful to avoid surgery in such cases.

\section{ThyroSeq}

ThyroSeq represents one of the molecular approaches available for the evaluation of indeterminate thyroid nodules that is based on the detection of molecular 
alterations in cell DNA and RNA seen in thyroid cancer [64]. Its first and most rudimentary version was introduced into clinical practice in 2007 as a sevengene panel (ThyroSeq v0) [50] [65]. The next versions of the test drifted towards the next-generation sequencing platforms and included a 13-gene panel that was introduced in 2013 (ThyroSeq v1) [48] and a 56-gene panel inaugurated in 2014 (ThyroSeq v2) [46] [47]. The ThyroSeq v2 version used DNA plus RNA for detecting point mutations/indels and gene fusions found in roughly $90 \%$ of PTC but also used a limited gene expression panel to monitor the cellularity and cell lineage of the samples [45]. This permitted an overall high positive predictive value (PPV) and negative predictive value (NPV) for the detection of cancer in indetermined nodules and aided the recognition of parathyroid glands and medullary thyroid carcinomas [47] [66]. Recent improvements in the molecular characteristics of less conventional types of thyroid cancer, particularly Hurthle cell carcinomas presented an opportunity for a more complete and accurate detection of all types of thyroid cancer, which led to the latest version of the ThyroSeq test [67] [68].

The latest version is the ThyroSeq $\mathrm{v}$ genomic classifier (GC) that was launched for clinical use at the end of 2017/beginning of 2018 [64]. This test uses nextgeneration sequencing technology expanded to analyze 112 genes, offering data from more than 12,000 mutational hotspots and more than 150 gene fusion types [69]. It reveals five diverse classes of genetic alterations: 1) mutations (single nucleotide variants); 2) insertions and deletions; 3) gene fusions; 4) gene expression alterations; and 5) copy number alterations [64]. Compared with the ThyroSeq v2 version, the key improvements of the ThyroSeq v3 GC include the analysis of a larger number of genes, mutation hotspots, and gene fusions; the detection of DNA copy number alterations, which is particularly important for Hurthle cell and follicular carcinomas; and the use of a GC [69]. This GC is based on the algorithmic analysis of all identified genetic alterations and their level (mutant allele frequency) to report the results as negative (including currently negative) or positive, in so doing providing a more specific assessment of thyroid nodules for cancer probability with a positive test. The ThyroSeq v3 GC has undergone validation of its analytical performance in a study of 238 resected tissue samples and 175 FNA samples [69]. It has been adjusted to handle fresh thyroid FNA samples collected into nucleic acid preservative solution and for formalin-fixed and paraffin-embedded sections of thyroid tissue or cytology cell block [69].

The ThyroSeq v3 GC utilizes a registered Genomic Classifier (GC) based on the algorithmic analysis of all detected genetic alterations to report the test result as positive or negative. ThyroSeq test consists of several steps:

- It starts with the assessment of the FNA sample cellularity:

- This is a quality assurance (QA) step that establishes if the provided sample has enough cells to proceed with the analysis.

- If the number of cells is below the obligatory limit, the test is cancelled. 
- Next, cellular composition of the sample is appraised:

- This step guarantees that the provided sample has an adequate proportion of thyroid follicular cells.

- It also allows precise detection of C-cells (MTC), parathyroid cells, and other non-thyroidal cells.

- Subsequently, the generated next generation sequencing data on 112 genes are processed using an in-house bioinformatic pipeline that applies a complex algorithm to estimate cancer probability in the tested nodule:

- The algorithm was built based on cancer probability associated with each genetic alteration and their combination and validated in a prospective, multicenter, double-blind study.

- After that, the test results and findings are reviewed by a board-certified pathologist who verifies all findings and releases the test report.

Steward et al in a prospective, double-blinded, multicenter study reported the results of the clinical validation of the ThyroSeq v3 GC in thyroid nodules with indeterminate FNA cytology [17]. The study recruited 782 patients with 1013 thyroid nodules at nine institutions in the United States and one institution in Singapore. The samples were collected by either rinsing the residual material in the aspiration needle from all passes or by placing a dedicated pass into the collection tube containing nucleic acid preservative solution. The study prerequisites were to have indeterminate or suspicious thyroid nodules (Bethesda III, IV, or V) with informative ThyroSeq results, and a definitive surgical pathology result. Just 257 samples met all study inclusion criteria. The surgical pathology results were assessed centrally by a panel of expert thyroid pathologists. On the other hand, by study design, cytology smears were not evaluated centrally so that the study could represent "real-world" experience of thyroid nodules diagnosed as indeterminate cytology. The study was double-blinded, with molecular testing personnel were blinded to all FNA and histopathology diagnoses, and both cytologists and pathologists were blinded to molecular testing results. In addition, pathologists were blinded to the diagnoses made by local and other panel pathologists. The discordant cases were evaluated by the panel to arrive at a consensus diagnosis. In the current study there was no post-unblind sample exclusion.

The primary outcome of the study was determining the sensitivity, specificity, NPV, and PPV of the ThyroSeq v3 GC in predicting the histopathologic diagnosis of a benign nodule versus cancer/noninvasive follicular thyroid neoplasm with papillary-like nuclear features (NIFTP) in thyroid nodules with indeterminate category III (AUS/FLUS) and category IV (SFN/FN) cytology results [17]. Although not considered as cancer, NIFTP is a precancerous, borderline tumor that requires surgical excision, and therefore it was grouped with cancer for data analysis [40].

The final study cohort included 154 thyroid nodules and 93 thyroid nodules diagnosed as AUS/FLUS and SFN/FN, respectively. The ThyroSeq V3 GC demonstrated a combined sensitivity of $94 \%$ and a specificity of $82 \%$ for cases diag- 
nosed as AUS/FLUS and SFN/FN [17]. With a $28 \%$ prevalence of cancer/NIFTP, the test demonstrated a NPV of $97 \%$ among these samples. There were only five false-negative test results in the entire study, representing four PTC and one minimally invasive follicular carcinoma. All the missed cancers were low-risk, intrathyroidal, and low-stage tumors with no vascular invasion, extrathyroidal extension, or other aggressive features.

The main use of ThyroSeq is to provide accurate cancer diagnosis in thyroid nodules with indeterminate FNA cytology. Indeterminate risk of malignancy in thyroid nodules with indeterminate FNA cytology (Bethesda III, IV, and V) hinders clinical management of these patients. ThyroSeq stratifies these thyroid nodules into those that are likely benign and may be observed and those that are likely malignant and require surgical treatment. For the latter nodules, ThyroSeq may provide additional information to help surgeons decide the extent of surgical management (lobectomy vs. total thyroidectomy). In addition, ThyroSeq may help to clarify the diagnosis in benign cytology nodules (Bethesda II) with clinical suspicion for malignancy. ThyroSeq detects chromosomal copy number alterations, which are a hallmark of Hürthle cell cancer [67] [68]. Data from the multicenter clinical validation study and an independent real-world study show reliable stratification of Hürthle cell nodules with as high as $100 \%$ sensitivity, $67 \%$ specificity, $100 \%$ negative predictive value (NPV) and $64 \%$ positive predictive value (PPV) [17] [70].

Based on the ATA guidelines, thyroid cancer risk stratification is imperative for selecting the appropriate extent of surgery (lobectomy vs total thyroidectomy), radioactive iodine (RAI) treatment, and intensity of follow-up [7]. Most thyroid cancers are indolent in nature, and these patients are at low risk for disease recurrence after adequate management. These patients can be treated by thyroid lobectomy and are unlikely to benefit from RAI ablation and TSH suppression. Conversely, patients with high-risk neoplasms would benefit from up-front total thyroidectomy, which facilitates post-operative RAI administration and disease monitoring [7]. Even though the role of using molecular testing to guide treatment decisions beyond the primary management of thyroid nodules is undetermined, ongoing research is working to increase our understanding and ability to further risk stratify thyroid cancer and possibly guide early treatment decisions, including the extent of surgery. ThyroSeq may provide a complete pre-operative assessment of the risk of cancer recurrence in patients with thyroid nodules.

ThyroSeq v3 Cancer Risk Classifier (CRC) is being developed to be used in FNAs from cytologically malignant (Bethesda VI) nodules and in resected thyroid cancers (formalin fixed paraffin embedded (FFPE)) to help in cancer risk stratification and patient management. Analogous to ThyroSeq v3 Genomic Classifier which is used for nodules with indeterminate cytology (Bethesda III to V), it uses a next-generation sequencing of DNA and RNA from 112 genes. Yet, instead of predicting of probability of cancer in a thyroid nodule, the main objective of the test is to predict risk of cancer recurrence that is required for se- 
lecting the extent of surgery and further adjuvant interventions for patients with thyroid cancer. The findings are reported as Low (RAS-like alterations, low-risk CNA), Intermediate (BRAF-like alterations, intermediate-risk CNA) or High risk (TERT and other high-risk mutations, or multiple mutations) of Cancer Recurrence [71].

ThyroSeq offers the ability to evaluate the Risk of Cancer Recurrence (RCR) based on the detection of all known molecular alterations associated with thyroid cancer aggressiveness (TERT, TP53, AKT1, PIK3CA, among others) [69], a case-control study of 287 patients with distant metastasis (DM) or without DM with a 5-years of follow-up or more that found that genetic profiling using this molecular assay provided accurate and robust risk stratification for DM in patients with differentiated thyroid cancer (DTC), based on prognostic information contained in the proprietary database of more than 3000 thyroid nodules with known surgical outcome, and the assessment of cancer risk and evaluation of aggressive thyroid cancers in studies that utilized ThyroSeq [71] [72] [73].

ThyroSeq offers the ability to detect the therapeutic targets for FDA approved drugs or enrollment into clinical trials in patients with advanced thyroid cancer [69] [74] [75] [76] [77]. Each ThyroSeq report contains information matching the detected gene target with corresponding treatment options. For ATC (anaplastic thyroid carcinoma), ThyroSeq provides rapid detection of the BRAF V600E mutation critical for effective treatment.

\section{Conclusions}

The research of molecular testing in thyroid nodules and thyroid cancer is progressing very rapidly, but thoughtfulness must be used when incorporating molecular testing for thyroid nodules into clinical practice. A clear appreciation of the goals and restraints of molecular testing must be integrated into how physicians use and explain molecular testing to patients. Molecular tests can help rule in cancer for indeterminate thyroid nodules with very specific mutations for thyroid cancer, such as BRAF and RET/PTC, and can help reduce the rates of completion thyroidectomies in this era of de-escalation of the management of thyroid disease. The PPV of malignant cytology (Bethesda VI) is $98 \%$; and even though molecular testing improves specificity and PPV, it falls short of this ideal for other mutations.

Integration of molecular tests into the management algorithm for indeterminate thyroid nodules is now encouraged by many important international guidelines. Even though considerable research exists on the diagnostic performance of commercial molecular tests, there are some major imperfections to their application, interpretation, clinical impact, and cost which should be addressed before their routine use. Like other diagnostic tests, these studies are best used in a thoughtful manner and in a case-by-case basis for thyroid nodules with indeterminate diagnosis as recommended by professional societies such as the American Thyroid Association and American Association for Endocrine Surgeons. Mo- 
lecular testing should only be used if the result of a test would otherwise alter the recommended treatment.

\section{Disclosures}

All the authors involved in the elaboration of this manuscript do not have any disclosures of any commercial interest, financial or material support.

\section{Conflicts of Interest}

The authors declare no conflicts of interest regarding the publication of this paper.

\section{References}

[1] Seib, C.D. and Sosa, J.A. (2019) Evolving Understanding of the Epidemiology of Thyroid Cancer. Endocrinology and Metabolism Clinics of North America, 48, 23-35. https://doi.org/10.1016/j.ecl.2018.10.002

[2] Prete, A., et al. (2020) Update on Fundamental Mechanisms of Thyroid Cancer. Frontiers in Endocrinology (Lausanne), 11, 102. https://doi.org/10.3389/fendo.2020.00102

[3] Takano, T. (2014) Fetal Cell Carcinogenesis of the Thyroid: A Modified Theory Based on Recent Evidence. Endocrine Journal, 61, 311-320. https://doi.org/10.1507/endocrj.EJ13-0517

[4] Mazzaferri, E.L. (1999) An Overview of the Management of Papillary and Follicular Thyroid Carcinoma. Thyroid, 9, 421-427. https://doi.org/10.1089/thy.1999.9.421

[5] Guth, S., et al. (2009) Very High Prevalence of Thyroid Nodules Detected by High Frequency (13 MHz) Ultrasound Examination. European Journal of Clinical Investigation, 39, 699-706. https://doi.org/10.1111/j.1365-2362.2009.02162.x

[6] Arrangoiz, R., Cordera, F., Caba, D., Moreno, E., de Leon, E.L. and Munoz, M. (2018) Management Approach to Thyroid Nodules. International Journal of Otolaryngology and Head \& Neck Surgery, 7, 214-227. https://doi.org/10.4236/ijohns.2018.74023

[7] Haugen, B.R., et al. (2016) 2015 American Thyroid Association Management Guidelines for Adult Patients with Thyroid Nodules and Differentiated Thyroid Cancer: The American Thyroid Association Guidelines Task Force on Thyroid Nodules and Differentiated Thyroid Cancer. Thyroid, 26, 1-133.

https://doi.org/10.1089/thy.2015.0020

[8] Gharib, H. and Papini, E. (2007) Thyroid Nodules: Clinical Importance, Assessment, and Treatment. Endocrinology and Metabolism Clinics of North America, 36, 707735. https://doi.org/10.1016/j.ecl.2007.04.009

[9] Patel, K.N., et al. (2020) The American Association of Endocrine Surgeons Guidelines for the Definitive Surgical Management of Thyroid Disease in Adults. Annals of Surgery, 271, e21-e93.

[10] Grant, E.G., et al. (2015) Thyroid Ultrasound Reporting Lexicon: White Paper of the ACR Thyroid Imaging, Reporting and Data System (TIRADS) Committee. Journal of the American College of Radiology, 12, 1272-1279. https://doi.org/10.1016/j.jacr.2015.07.011

[11] Arrangoiz, R., Cordera, F., Caba, D., Moreno, E., Luque-de-Leon, E. and Muñoz, M. (2019) Thyroid Cancer. International Journal of Otolaryngology and Head \& Neck 
Surgery, 8, 217-270. https://doi.org/10.4236/ijohns.2019.86024

[12] Cibas, E.S. and Ali, S.Z. (2009) The Bethesda System for Reporting Thyroid Cytopathology. Thyroid, 19, 1159-1165. https://doi.org/10.1089/thy.2009.0274

[13] Cibas, E.S. and Ali, S.Z. (2017) The 2017 Bethesda System for Reporting Thyroid Cytopathology. Thyroid, 27, 1341-1346. https://doi.org/10.1089/thy.2017.0500

[14] Febbo, P.G., et al. (2011) NCCN Task Force Report: Evaluating the Clinical Utility of Tumor Markers in Oncology. Journal of the National Comprehensive Cancer Network, 9, S1-S32. https://doi.org/10.6004/jnccn.2011.0137

[15] Sahli, Z.T., et al. (2018) Preoperative Molecular Markers in Thyroid Nodules. Frontiers in Endocrinology (Lausanne), 9, 179. https://doi.org/10.3389/fendo.2018.00179

[16] Mayson, S.E. and Haugen, B.R. (2019) Molecular Diagnostic Evaluation of Thyroid Nodules. Endocrinology and Metabolism Clinics of North America, 48, 85-97. https://doi.org/10.1016/j.ecl.2018.10.004

[17] Steward, D.L., et al. (2019) Performance of a Multigene Genomic Classifier in Thyroid Nodules with Indeterminate Cytology: A Prospective Blinded Multicenter Study. JAMA Oncology, 5, 204-212. https://doi.org/10.1001/jamaoncol.2018.4616

[18] Patel, K.N., et al. (2018) Performance of a Genomic Sequencing Classifier for the Preoperative Diagnosis of Cytologically Indeterminate Thyroid Nodules. JAMA Surgery, 153, 817-824. https://doi.org/10.1001/jamasurg.2018.1153

[19] Yip, L. and Sosa, J.A. (2016) Molecular-Directed Treatment of Differentiated Thyroid Cancer: Advances in Diagnosis and Treatment. JAMA Surgery, 151, 663-670. https://doi.org/10.1001/jamasurg.2016.0825

[20] Eszlinger, M., et al. (2017) Evaluation of a Two-Year Routine Application of Molecular Testing of Thyroid Fine-Needle Aspirations Using a Seven-Gene Panel in a Primary Referral Setting in Germany. Thyroid, 27, 402-411. https://doi.org/10.1089/thy.2016.0445

[21] Kuo, J.H., et al. (2019) Updates in the Management of Thyroid Nodules. Current Problems in Surgery, 56, 103-127. https://doi.org/10.1067/j.cpsurg.2018.12.003

[22] Gharib, H., et al. (2016) American Association of Clinical Endocrinologists, American College of Endocrinology, and Associazione Medici Endocrinologi Medical Guidelines for Clinical Practice for the Diagnosis and Management of Thyroid Nodules-2016 Update. Endocrine Practice, 22, 622-639. https://doi.org/10.4158/EP161208.GL

[23] Patel, K.N., et al. (2020) Executive Summary of the American Association of Endocrine Surgeons Guidelines for the Definitive Surgical Management of Thyroid Disease in Adults. Annals of Surgery, 271, 399-410. https://doi.org/10.1097/SLA.0000000000003735

[24] Nikiforov, Y.E. (2011) Molecular Analysis of Thyroid Tumors. Modern Pathology, 24, S34-S43. https://doi.org/10.1038/modpathol.2010.167

[25] Adeniran, A.J., et al. (2006) Correlation between Genetic Alterations and Microscopic Features, Clinical Manifestations, and Prognostic Characteristics of Thyroid Papillary Carcinomas. The American Journal of Surgical Pathology, 30, 216-222. https://doi.org/10.1097/01.pas.0000176432.73455.1b

[26] Kimura, E.T., et al. (2003) High Prevalence of BRAF Mutations in Thyroid Cancer: Genetic Evidence for Constitutive Activation of the RET/PTC-RAS-BRAF Signaling Pathway in Papillary Thyroid Carcinoma. Cancer Research, 63, 1454-1457.

[27] Soares, P., et al. (2003) BRAF Mutations and RET/PTC Rearrangements Are Alternative Events in the Etiopathogenesis of PTC. Oncogene, 22, 4578-4580. 
https://doi.org/10.1038/sj.onc.1206706

[28] Frattini, M., et al. (2004) Alternative Mutations of BRAF, RET and NTRK1 Are Associated with Similar but Distinct Gene Expression Patterns in Papillary Thyroid Cancer. Oncogene, 23, 7436-7440. https://doi.org/10.1038/sj.onc.1207980

[29] Nikiforova, M.N., et al. (2003) RAS Point Mutations and PAX8-PPAR Gamma Rearrangement in Thyroid Tumors: Evidence for Distinct Molecular Pathways in Thyroid Follicular Carcinoma. The Journal of Clinical Endocrinology \& Metabolism, 88, 2318-2326. https://doi.org/10.1210/jc.2002-021907

[30] Garcia-Rostan, G., et al. (2005) Mutation of the PIK3CA Gene in Anaplastic Thyroid Cancer. Cancer Research, 65, 10199-10207.

https://doi.org/10.1158/0008-5472.CAN-04-4259

[31] Hou, P., et al. (2007) Genetic Alterations and Their Relationship in the Phosphatidylinositol 3-Kinase/Akt Pathway in Thyroid Cancer. Clinical Cancer Research, 13, 1161-1170. https://doi.org/10.1158/1078-0432.CCR-06-1125

[32] Ricarte-Filho, J.C., et al. (2009) Mutational Profile of Advanced Primary and Metastatic Radioactive Iodine-Refractory Thyroid Cancers Reveals Distinct Pathogenetic Roles for BRAF, PIK3CA, and AKT1. Cancer Research, 69, 4885-4893.

https://doi.org/10.1158/0008-5472.CAN-09-0727

[33] Kondo, T., Ezzat, S. and Asa, S.L. (2006) Pathogenetic Mechanisms in Thyroid Follicular-Cell Neoplasia. Nature Reviews Cancer, 6, 292-306. https://doi.org/10.1038/nrc1836

[34] Pierotti, M.A., et al. (1996) Cytogenetics and Molecular Genetics of Carcinomas Arising from Thyroid Epithelial Follicular Cells. Genes Chromosomes Cancer, 16, $1-14$. https://doi.org/10.1002/(SICI)1098-2264(199605)16:1<1::AID-GCC1>3.0.CO;2-4

[35] Bongarzone, I., et al. (1998) RET/NTRK1 Rearrangements in Thyroid Gland Tumors of the Papillary Carcinoma Family: Correlation with Clinicopathological Features. Clinical Cancer Research, 4, 223-228.

[36] Cooper, D.S., et al. (2006) Management Guidelines for Patients with Thyroid Nodules and Differentiated Thyroid Cancer. Thyroid, 16, 109-142. https://doi.org/10.1089/thy.2006.16.109

[37] Roth, M.Y., Witt, R.L. and Steward, D.L. (2018) Molecular Testing for Thyroid Nodules: Review and Current State. Cancer, 124, 888-898.

https://doi.org/10.1002/cncr.30708

[38] Cibas, E.S., et al. (2013) A Prospective Assessment Defining the Limitations of Thyroid Nodule Pathologic Evaluation. Annals of Internal Medicine, 159, 325-332. https://doi.org/10.7326/0003-4819-159-5-201309030-00006

[39] Sacks, W.L., et al. (2016) Impact of Afirma Gene Expression Classifier on Cytopathology Diagnosis and Rate of Thyroidectomy. Cancer Cytopathology, 124, 722-728. https://doi.org/10.1002/cncy.21749

[40] Nikiforov, Y.E., et al. (2016) Nomenclature Revision for Encapsulated Follicular Variant of Papillary Thyroid Carcinoma: A Paradigm Shift to Reduce Overtreatment of Indolent Tumors. JAMA Oncology, 2, 1023-1029. https://doi.org/10.1001/jamaoncol.2016.0386

[41] Baloch, Z.W., et al. (2008) Diagnostic Terminology and Morphologic Criteria for Cytologic Diagnosis of Thyroid Lesions: A Synopsis of the National Cancer Institute Thyroid Fine-Needle Aspiration State of the Science Conference. Diagnostic Cytopathology, 36, 425-437. https://doi.org/10.1002/dc.20830

[42] Wang, H.H., et al. (2010) Suspicious for Malignancy. In: S.Z. Ali, Cibas, E.S., Eds., 
The Bethesda System for Reporting Thyroid Cytopathology, Springer Science + Business Media, Berlin. https://doi.org/10.1007/978-0-387-87666-5 7

[43] Walsh, P.S., et al. (2012) Analytical Performance Verification of a Molecular Diagnostic for Cytology-Indeterminate Thyroid Nodules. The Journal of Clinical Endocrinology \& Metabolism, 97, E2297-E2306. https://doi.org/10.1210/jc.2012-1923

[44] Alexander, E.K., et al. (2012) Preoperative Diagnosis of Benign Thyroid Nodules with Indeterminate Cytology. The New England Journal of Medicine, 367, 705-715. https://doi.org/10.1056/NEJMoa1203208

[45] Cancer Genome Atlas Research, N. (2014) Integrated Genomic Characterization of Papillary Thyroid Carcinoma. Cell, 159, 676-690.

https://doi.org/10.1016/j.cell.2014.09.050

[46] Nikiforov, Y.E., et al. (2014) Highly Accurate Diagnosis of Cancer in Thyroid Nodules with Follicular Neoplasm/Suspicious for a Follicular Neoplasm Cytology by ThyroSeq v2 Next-Generation Sequencing Assay. Cancer, 120, 3627-3634. https://doi.org/10.1002/cncr.29038

[47] Nikiforov, Y.E., et al. (2015) Impact of the Multi-Gene ThyroSeq Next-Generation Sequencing Assay on Cancer Diagnosis in Thyroid Nodules with Atypia of Undetermined Significance/Follicular Lesion of Undetermined Significance Cytology. Thyroid, 25, 1217-1223. https://doi.org/10.1089/thy.2015.0305

[48] Nikiforova, M.N., Wald, A.I., Roy, S., Durso, M.B. and Nikiforov, Y.E. (2013) Targeted Next-Generation Sequencing Panel (ThyroSeq) for Detection of Mutations in Thyroid Cancer. The Journal of Clinical Endocrinology \& Metabolism, 98, E1852E1860. https://doi.org/10.1210/jc.2013-2292

[49] Benjamin, H., et al. (2016) Analytical Validity of a microRNA-Based Assay for Diagnosing Indeterminate Thyroid FNA Smears from Routinely Prepared Cytology Slides. Cancer Cytopathology, 124, 711-721. https://doi.org/10.1002/cncy.21731

[50] Nikiforov, Y.E., et al. (2011) Impact of Mutational Testing on the Diagnosis and Management of Patients with Cytologically Indeterminate Thyroid Nodules: A Prospective Analysis of 1056 FNA Samples. The Journal of Clinical Endocrinology \& Metabolism, 96, 3390-3397. https://doi.org/10.1210/jc.2011-1469

[51] Najafian, A., et al. (2017) RAS Mutations, and RET/PTC and PAX8/PPAR-Gamma Chromosomal Rearrangements Are Also Prevalent in Benign Thyroid Lesions: Implications Thereof and A Systematic Review. Thyroid, 27, 39-48.

https://doi.org/10.1089/thy.2016.0348

[52] Armstrong, M.J., et al. (2014) PAX8/PPARgamma Rearrangement in Thyroid Nodules Predicts Follicular-Pattern Carcinomas, in Particular the Encapsulated Follicular Variant of Papillary Carcinoma. Thyroid, 24, 1369-1374. https://doi.org/10.1089/thy.2014.0067

[53] Nishino, M. (2016) Molecular Cytopathology for Thyroid Nodules: A Review of Methodology and Test Performance. Cancer Cytopathology, 124, 14-27. https://doi.org/10.1002/cncy.21612

[54] Xing, M., Haugen, B.R. and Schlumberger, M. (2013) Progress in Molecular-Based Management of Differentiated Thyroid Cancer. The Lancet, 381, 1058-1069. https://doi.org/10.1016/S0140-6736(13)60109-9

[55] Kloos, R.T. (2017) Molecular Profiling of Thyroid Nodules: Current Role for the Afirma Gene Expression Classifier on Clinical Decision Making. Molecular Imaging and Radionuclide Therapy, 26, 36-49. https://doi.org/10.4274/2017.26.suppl.05

[56] Cantara, S., et al. (2010) Impact of Proto-Oncogene Mutation Detection in Cytological Specimens from Thyroid Nodules Improves the Diagnostic Accuracy of Cy- 
tology. The Journal of Clinical Endocrinology \& Metabolism, 95, 1365-1369. https://doi.org/10.1210/jc.2009-2103

[57] Eszlinger, M., et al. (2014) Impact of Molecular Screening for Point Mutations and Rearrangements in Routine Air-Dried Fine-Needle Aspiration Samples of Thyroid Nodules. Thyroid, 24, 305-313. https://doi.org/10.1089/thy.2013.0278

[58] Eszlinger, M., et al. (2015) Molecular Testing of Thyroid Fine-Needle Aspirations Improves Presurgical Diagnosis and Supports the Histologic Identification of Minimally Invasive Follicular Thyroid Carcinomas. Thyroid, 25, 401-409. https://doi.org/10.1089/thy.2014.0362

[59] Rossi, M., et al. (2015) Relevance of BRAF(V600E) Mutation Testing versus RAS Point Mutations and RET/PTC Rearrangements Evaluation in the Diagnosis of Thyroid Cancer. Thyroid, 25, 221-228. https://doi.org/10.1089/thy.2014.0338

[60] Moses, W., et al. (2010) Molecular Testing for Somatic Mutations Improves the Accuracy of Thyroid Fine-Needle Aspiration Biopsy. World Journal of Surgery, 34, 2589-2594. https://doi.org/10.1007/s00268-010-0720-0

[61] Krane, J.F., et al. (2015) Molecular Analysis of Residual ThinPrep Material from Thyroid FNAs Increases Diagnostic Sensitivity. Cancer Cytopathology, 123, 356-361. https://doi.org/10.1002/cncy.21546

[62] Pagan, M., et al. (2016) The Diagnostic Application of RNA Sequencing in Patients with Thyroid Cancer: An Analysis of 851 Variants and 133 Fusions in 524 Genes. BMC Bioinformatics, 17, 6. https://doi.org/10.1186/s12859-015-0849-9

[63] Chudova, D., et al. (2010) Molecular Classification of Thyroid Nodules Using HighDimensionality Genomic Data. The Journal of Clinical Endocrinology \& Metabolism, 95, 5296-5304. https://doi.org/10.1210/jc.2010-1087

[64] Nikiforov, Y.E. and Baloch, Z.W. (2019) Clinical Validation of the ThyroSeq v3 Genomic Classifier in Thyroid Nodules with Indeterminate FNA Cytology. Cancer Cytopathology, 127, 225-230. https://doi.org/10.1002/cncy.22112

[65] Nikiforov, Y.E., et al. (2009) Molecular Testing for Mutations in Improving the Fine-Needle Aspiration Diagnosis of Thyroid Nodules. The Journal of Clinical Endocrinology \& Metabolism, 94, 2092-2098. https://doi.org/10.1210/jc.2009-0247

[66] Cho, M., et al. (2017) Distinguishing Parathyroid and Thyroid Lesions on Ultrasound-Guided Fine-Needle Aspiration: A Correlation of Clinical Data, Ancillary Studies, and Molecular Analysis. Cancer Cytopathology, 125, 674-682.

https://doi.org/10.1002/cncy.21888

[67] Ganly, I., et al. (2018) Integrated Genomic Analysis of Hurthle Cell Cancer Reveals Oncogenic Drivers, Recurrent Mitochondrial Mutations, and Unique Chromosomal Landscapes. Cancer Cell, 34, 256-270e5. https://doi.org/10.1016/j.ccell.2018.07.002

[68] Gopal, R.K., et al. (2018) Widespread Chromosomal Losses and Mitochondrial DNA Alterations as Genetic Drivers in Hurthle Cell Carcinoma. Cancer Cell, 34, 242$255 \mathrm{e} 5$.

[69] Nikiforova, M.N., et al. (2018) Analytical Performance of the ThyroSeq v3 Genomic Classifier for Cancer Diagnosis in Thyroid Nodules. Cancer, 124, 1682-1690. https://doi.org/10.1002/cncr.31245

[70] Schatz-Siemers, N., et al. (2019) Hurthle Cell Lesions on Thyroid Fine Needle Aspiration Cytology: Molecular and Histologic Correlation. Diagnostic Cytopathology, 47, 977-985. https://doi.org/10.1002/dc.24247

[71] Chin, P.D., et al. (2020) Correlation of ThyroSeq Results with Surgical Histopathology in Cytologically Indeterminate Thyroid Nodules. Endocrine Pathology, 31, 377 - 
384. https://doi.org/10.1007/s12022-020-09641-2

[72] Yip, L., et al. (2015) Tumor Genotype Determines Phenotype and Disease-Related Outcomes in Thyroid Cancer: A Study of 1510 Patients. Annals of Surgery, 262, 519-525. https://doi.org/10.1097/SLA.0000000000001420

[73] Chernock, R.D., et al. (2020) Poorly Differentiated Thyroid Carcinoma of Childhood and Adolescence: A Distinct Entity Characterized by DICER1 Mutations. Modern Pathology, 33, 1264-1274. https://doi.org/10.1038/s41379-020-0458-7

[74] Panebianco, F., et al. (2019) Characterization of Thyroid Cancer Driven by Known and Novel ALK Fusions. Endocrine-Related Cancer, 26, 803-814. https://doi.org/10.1530/ERC-19-0325

[75] Seethala, R.R., et al. (2017) Clinical and Morphologic Features of ETV6-NTRK3 Translocated Papillary Thyroid Carcinoma in an Adult Population without Radiation Exposure. The American Journal of Surgical Pathology, 41, 446-457. https://doi.org/10.1097/PAS.0000000000000814

[76] Kelly, L.M., et al. (2014) Identification of the Transforming STRN-ALK Fusion as a Potential Therapeutic Target in the Aggressive Forms of Thyroid Cancer. Proceedings of the National Academy of Sciences of the United States of America, 111, 4233 4238. https://doi.org/10.1073/pnas.1321937111

[77] Nikiforov, Y.E. (2002) RET/PTC Rearrangement in Thyroid Tumors. Endocrine Pathology, 13, 3-16. https://doi.org/10.1385/EP:13:1:03 